\title{
Modelagem matemática e difusividade efetiva de folhas de aroeira durante a secagem ${ }^{1}$
}

\author{
André Luís Duarte Goneli², Maria do Carmo Vieira², \\ Henrique da Cruz Benitez Vilhasanti², Alexandre Alves Gonçalves ${ }^{2}$
}

\begin{abstract}
Mathematical modeling and effective diffusion of Schinus terebinthifolius leaves during drying

The drying process of agricultural products is extensively used worldwide for controlling and maintaining their quality. For medicinal and aromatic plants, this importance increases even more. Thus, this study aimed at evaluating the drying kinetics of Schinus terebinthifolius Raddi leaves, as well as adjusting different mathematical models to the experimental values of moisture ratio. The leaves were harvested with initial moisture content of approximately $65 \%$ (w.b.) and submitted to the drying process under controlled conditions of temperature $\left(40^{\circ} \mathrm{C}, 50^{\circ} \mathrm{C}, 60^{\circ} \mathrm{C}\right.$ and $\left.70^{\circ} \mathrm{C}\right)$, up to the approximate moisture content of $10 \%$ (w.b.). Six mathematical models were adjusted to the experimental data cited at the specific literature and used to predict the drying process of agricultural products. According to the results obtained, it was concluded that the modified Henderson \& Pabis and Midilli models were the ones that best represented the drying kinetics of $S$. terebinthifolius leaves. The temperature increase of the drying air promoted a higher rate of water removal from the product. The effective diffusion coefficient increased with the temperature elevation, and its relation to the drying temperature fitted the Arrhenius equation, which presented activation energy for the liquid diffusion, during the drying process, of $74.96 \mathrm{~kJ} \mathrm{~mol}^{-1}$, for $S$. terebinthifolius leaves.
\end{abstract}

KEY-WORDS: Midilli model; moisture ratio; activation energy.

\section{INTRODUÇÃO}

Plantas medicinais e aromáticas são a principal matéria-prima de grande quantidade de indústrias de alimentos, cosmética e farmacêutica. Seu uso é bastante disseminado entre a população brasileira, sendo vários os fatores que colaboram para o desenvolvimento de práticas de saúde que incluam plantas medicinais, dentre eles o baixo custo da utilização da medicina caseira e as propriedades terapêuticas

\section{RESUMO}

A secagem de produtos agrícolas é largamente utilizada no mundo, para o controle e a manutenção da sua qualidade. Em se tratando de plantas medicinais e aromáticas, esta importância cresce ainda mais. Assim, o presente trabalho objetivou avaliar a cinética de secagem de folhas de aroeira (Schinus terebinthifolius Raddi), bem como ajustar diferentes modelos matemáticos aos valores experimentais de razão de umidade. As folhas foram colhidas com teor de água inicial de $65 \%$ (b.u.), sendo submetidas à secagem sob condições controladas de temperatura $\left(40^{\circ} \mathrm{C}, 50^{\circ} \mathrm{C}, 60^{\circ} \mathrm{C}\right.$ e $\left.70^{\circ} \mathrm{C}\right)$, até o teor de água aproximado de $10 \%$ (b.u.). Seis modelos matemáticos foram ajustados aos dados experimentais citados na literatura específica e utilizados para a representação do processo de secagem de produtos agrícolas. Com base nos resultados obtidos, pôde-se concluir que os modelos de Henderson \& Pabis modificado e Midilli foram os que melhor representaram a cinética de secagem das folhas de aroeira. O aumento da temperatura do ar de secagem promoveu maior taxa de remoção de água do produto. O coeficiente de difusão efetivo aumentou com a elevação da temperatura, e sua relação com a temperatura de secagem pôde ser descrita pela equação de Arrhenius, que apresentou energia de ativação para a difusão líquida, durante a secagem, de $74,96 \mathrm{~kJ} \mathrm{~mol}^{-1}$, para as folhas de aroeira.

PALAVRAS-CHAVE: Modelo de Midilli; razão de umidade; energia de ativação.

provenientes dos princípios ativos. Para atingir estes objetivos, a produção de fitoterápicos requer, necessariamente, estudos prévios, relativos a aspectos botânicos, agronômicos, fitoquímicos, farmacológicos e toxicológicos de desenvolvimento de metodologias analíticas e tecnológicas (Miguel \& Miguel 2004).

Considerando-se os benefícios da utilização de plantas medicinais, surge a necessidade de se estudar as vantagens do processo de secagem das mesmas, visando a uma melhor qualidade do produto final.

1. Trabalho recebido em maio/2013 e aceito para publicação em fev./2014 (nº registro: PAT 24384).

2. Universidade Federal da Grande Dourados (UFGD), Faculdade de Ciências Agrárias, Dourados, MS, Brasil. E-mails: andregoneli@ufgd.edu.br,mariavieira@ufgd.edu.br, henrique_vilhasanti716@hotmail.com, alexandre_alvesg@hotmail.com. 
Principal matéria-prima para a obtenção de produtos fitoterápicos, a parte aérea das plantas medicinais, normalmente, é colhida com elevado teor de água, sendo este o principal responsável pela má conservação pós-colheita do produto, uma vez que a água é a principal responsável pelo aumento de atividades metabólicas e mudanças químicas e físicas que ocorrem no produto, durante o seu armazenamento. Assim, objetivando a manutenção de sua qualidade após a colheita, é fundamental que as plantas medicinais tenham seu teor de água reduzido, sendo a secagem o processo mais utilizado para esta finalidade.

A secagem consiste em um complexo processo, que envolve a transferência de calor e massa entre $\mathrm{o}$ ar de secagem e o produto a ser seco, na qual o aumento da temperatura provoca o aumento da pressão parcial de vapor no produto, provocando a redução no teor de água. $O$ processo de secagem em espécies medicinais é uma operação de fundamental importância para o armazenamento, uma vez que a indústria farmacêutica de fitoterápicos não tem estrutura para utilizar plantas frescas nas quantidades exigidas para a produção industrial (Lorenzi \& Matos 2002). As condições de secagem e armazenamento são muito importantes para manter a qualidade destes produtos, sendo que as plantas medicinais e aromáticas devem ser submetidas imediatamente à secagem, após a colheita (Soysal \& Öztekin 1999).

A maioria dos estudos relacionados à secagem de produtos agrícolas se refere ao efeito de diversas variáveis externas, como a temperatura do ar, umidade relativa e velocidade do ar, dentre outras, sobre o produto. Para tanto, modelos matemáticos são utilizados para representar a cinética de secagem, frente a estas condições, sendo estes fundamentais para as simulações matemáticas de secagem, em camadas espessas de produto, que irão auxiliar no projeto de novos secadores ou no aperfeiçoamento de secadores já em operação (Berbert et al. 1995).

Existem três tipos de modelos de secagem utilizados para descrever a cinética de secagem de produtos agrícolas: o modelo teórico, que considera apenas a resistência interna à transferência de calor e água entre o produto e o ar quente, e os modelos semiteórico e empírico, que consideram somente a resistência externa à temperatura e umidade relativa do ar de secagem (Midilli et al. 2002, Panchariya et al. 2002).

Os modelos semiteóricos são, geralmente, derivados da simplificação da segunda Lei de Fick, oferecendo facilidade de uso, mas sendo válidos somente para as faixas de temperatura, umidade relativa, velocidade do ar e teor de água em que eles foram obtidos (Ozdemir \& Devres 1999, Panchariya et al. 2002).

Os modelos empíricos de secagem apresentam relação direta entre o teor de água do produto e o tempo de secagem. Eles negligenciam os fundamentos do processo de secagem, e seus parâmetros não têm qualquer significado físico. Desta forma, os modelos empíricos não fornecem uma perspectiva dos importantes processos que ocorrem durante a secagem, embora, em alguns casos, possam descrever as curvas de secagem, para determinadas condições experimentais (Keey 1972).

Dentre os modelos teóricos aplicados ao processo de secagem, o modelo da difusão é o mais intensamente investigado. Este modelo considera, como mecanismo principal, a difusão baseada na segunda Lei de Fick, que descreve que o fluxo de massa por unidade de área é proporcional ao gradiente de concentração de água (Park et al. 2002). A difusão em produtos agrícolas, durante a secagem, é um complexo processo, que pode envolver diferentes mecanismos, como a difusão molecular, capilar, na superfície, de vapor e térmica e o fluxo hidrodinâmico.

Uma vez que os modelos baseados na segunda Lei de Fick não são rigorosamente representativos para os diversos mecanismos que prevalecem no transporte de água em produtos agrícolas, o coeficiente de difusão determinado é considerado aparente ou efetivo (Roca et al. 2008). Outro fator que contribui para a complexidade do mecanismo de difusão é a diversidade da composição química e a estrutura física dos produtos. Desta forma, os dados disponíveis na literatura apresentam elevada variação nos seus valores, não só devido à complexidade dos produtos, como, também, em função dos diferentes métodos de estimação, tipo de material, teor de água, processo de secagem e metodologia utilizada para a sua obtenção (Zogzas et al. 1996).

Devido à importância da secagem de plantas medicinais, considerando-se a necessária conservação pós-colheita do produto, bem como a baixa capacidade de processamento de plantas úmidas, em quantidades exigidas para a produção industrial, a cinética de secagem de plantas medicinais tem sido estudada por diversos pesquisadores, para as mais diferentes espécies: parte aérea de Pectis brevipedunculata (Oliveira et al. 2013); folhas de Solanum lycocarpum (Prates et al. 2012); folhas de 
Ocimum basilicum L. (Reis et al. 2012); partes de plantas de Thymus vulgaris L. (Rocha et al. 2012); partes de plantas de Baccharis trimera (Radünz et al. 2011); folhas de Cymbopogon citratus (Martinazzo et al. 2007 e 2010); folhas de Moringa oleifera (Premi et al. 2010); e folhas de Mentha spicata L. (Doymaz 2006). Entretanto, não foram encontradas, na literatura, informações a respeito da cinética de secagem de folhas de aroeira.

Dessa forma, o presente trabalho foi desenvolvido com o objetivo de avaliar e ajustar diferentes modelos matemáticos aos dados de secagem de folhas de aroeira, bem como determinar o coeficiente de difusão efetivo, durante a secagem.

\section{MATERIAL E MÉTODOS}

O presente trabalho foi realizado no Laboratório de Propriedades Físicas de Produtos Agrícolas da Universidade Federal da Grande Dourados (UFGD), no município de Dourados (MS). Foram utilizadas folhas da parte aérea da aroeira (Schinus terebinthifolius), colhidas no Horto de Plantas Medicinais da Faculdade de Ciências Agrárias da Universidade Federal da Grande Dourados.

As folhas foram colhidas na primeira semana do mês de março de 2013, sempre nas primeiras horas da manhã, e sem ter ocorrido precipitação pluviométrica ou irrigação, a fim de evitar variação no teor de água inicial das mesmas. Após serem aleatoriamente colhidas, as folhas foram homogeneizadas, com a finalidade de se evitar qualquer tipo de interferência nos resultados, bem como retiradas as suas hastes. Para cada temperatura de secagem utilizada neste trabalho, foi realizada uma coleta de, aproximadamente, $300 \mathrm{~g}$ de folhas, em diferentes locais e plantas. Depois de feita a seleção e homogeneização, parte do material colhido foi destinado à determinação do teor de água e a outra parte (aproximadamente 25 g por repetição) colocada para secar. O teor de água das amostras foi determinado pelo método gravimétrico recomendado pela ASABE (2010), para forrageiras e plantas similares, em estufa com circulação forçada de ar a $103 \pm 1^{\circ} \mathrm{C}$, durante 24 horas.

A secagem do produto foi realizada para diferentes condições controladas de temperatura $\left(40^{\circ} \mathrm{C}\right.$, $50^{\circ} \mathrm{C}, 60^{\circ} \mathrm{C}$ e $70^{\circ} \mathrm{C}$ ), com suas correspondentes umidades relativas do ar de secagem $(20 \%, 12 \%, 7 \%$ e $3 \%$ ), durante a condução do experimento, em estufa com ventilação forçada, até que o produto atingisse o teor de água de equilíbrio.

O teor de água inicial das folhas de aroeira, no início dos tratamentos de secagem, foi de, aproximadamente, $65 \%$ (b.u.), sendo que, para fins de modelagem matemática, considerou-se o final da secagem quando o teor de água do produto estivesse próximo a 10\% (b.u.). Foram colocadas, no interior do equipamento, duas bandejas removíveis, com fundo telado, para permitir a passagem do ar através da camada de produto, sendo que, em cada bandeja, havia duas repetições de produto, totalizando, para cada temperatura de secagem utilizada, quatro repetições. A temperatura e a umidade relativa do ar foram monitoradas por meio de psicrômetro instalado próximo à estufa contendo as amostras.

Durante o processo de secagem, as bandejas com as amostras foram pesadas periodicamente. Foi utilizada uma balança digital de $0,001 \mathrm{~g}$ de resolução. O tempo entre as leituras foi controlado por meio da diferença de massas (conhecendo-se a massa e o teor de água inicial das folhas), de forma que não permitisse diferenças acentuadas de teor de água entre as leituras.

A razão de umidade (RU) das folhas da aroeira, durante a secagem nas diferentes condições de ar, foi determinada por meio da seguinte expressão:

$$
R U=\frac{M-M_{e}}{M_{i}-M_{e}}
$$

em que RU = razão de umidade do produto (adimensional); $\mathrm{M}=$ teor de água do produto (decimal, b.s.); $\mathrm{M}_{\mathrm{e}}=$ teor de água de equilíbrio do produto (decimal, b.s.); e $\mathrm{M}_{\mathrm{i}}=$ teor de água inicial do produto (decimal, b.s.).

Aos dados experimentais de razão de umidade, durante a secagem das folhas de aroeira, foram ajustados oito modelos matemáticos tradicionalmente utilizados para predizer o fenômeno da secagem de produtos agrícolas (Tabela 1).

O coeficiente de difusão efetivo das folhas de aroeira, para as diferentes condições de secagem $\left(40^{\circ} \mathrm{C}, 50^{\circ} \mathrm{C}, 60^{\circ} \mathrm{C}\right.$ e $\left.70^{\circ} \mathrm{C}\right)$, foi calculado utilizando-se a Equação 10, baseada na teoria da difusão líquida. Esta equação é a solução analítica para a segunda Lei de Fick, considerando-se a forma geométrica plana do produto e com aproximação de oito termos: 
Tabela 1. Modelos matemáticos utilizados para estimar as curvas de secagem.

\begin{tabular}{lll}
\hline \multicolumn{1}{c}{ Designação do modelo } & \multicolumn{1}{c}{ Modelo } \\
\hline Aproximação da difusão & $\mathrm{RU}=\mathrm{a} \exp (-\mathrm{k} \theta)+(1-\mathrm{a}) \exp (-\mathrm{k} \mathrm{b} \theta)$ & $(2)$ \\
Dois termos & $\mathrm{RU}=\mathrm{a} \exp (-\mathrm{k} \theta)+\mathrm{b} \exp \left(-\mathrm{k}_{1} \theta\right)$ & $(3)$ \\
Exponencial de dois termos & $\mathrm{RU}=\mathrm{a} \exp (-\mathrm{k} \theta)+(1-\mathrm{a}) \exp (-\mathrm{k} \mathrm{a} \theta)$ & $(4)$ \\
Henderson \& Pabis modificado & $\mathrm{RU}=\mathrm{a} \exp (-\mathrm{k} \theta)+\mathrm{b} \exp \left(-\mathrm{k}_{0} \theta\right)+\mathrm{c} \exp \left(-\mathrm{k}_{1} \theta\right)$ & $(5)$ \\
Logarítmico & $\mathrm{RU}=\mathrm{a} \exp (-\mathrm{k} \theta)+\mathrm{c}$ & $(6)$ \\
Midilli & $\mathrm{RU}=\mathrm{a} \exp \left(-\mathrm{k} \theta^{\mathrm{n}}\right)+\mathrm{b} \theta$ & $(7)$ \\
Page & $\mathrm{RU}=\exp \left(-\mathrm{k} \theta^{\mathrm{n}}\right)$ & $(9)$ \\
Thompson & $\mathrm{RU}=\exp \left\{\left[-\mathrm{a}-\left(\mathrm{a}^{2}+4 \mathrm{~b} \theta\right)^{0,5}\right] / 2 \mathrm{~b}\right\}$ & \\
\hline$\theta$ : tempo de secagem $(\mathrm{s}) ; \mathrm{k}, \mathrm{k}_{0}, \mathrm{k}_{\mathrm{t}}$ : constantes de secagem $\left(\mathrm{s}^{-1}\right) ; \mathrm{a}, \mathrm{b}, \mathrm{c}: \operatorname{coeficientes~dos~modelos.~}$
\end{tabular}

$R U=\frac{M-M_{e}}{M_{i}-M_{e}}=\frac{8}{\pi^{2}} \sum_{n=o}^{\infty} \frac{1}{(2 n+1)^{2}} \exp \left[-(2 n+1)^{2} \pi^{2} D_{i} \frac{t}{4 L^{2}}\right]$

em que $\mathrm{D}_{\mathrm{i}}=$ coeficiente de difusão efetivo $\left(\mathrm{m}^{2} \mathrm{~s}^{-1}\right)$; $\mathrm{L}=$ espessura do produto $(\mathrm{m}) ; \mathrm{t}=$ tempo de secagem (s); $\mathrm{n}=$ número de termos do modelo.

Para determinação da espessura das folhas de aroeira, foram realizadas 50 repetições por dia de coleta de material, utilizando-se paquímetro digital com resolução de $0,01 \mathrm{~mm}$. Para cada folha medida, eram feitas três medições, em diferentes locais. Foi calculada a média dos valores, obtendo-se $0,3631 \mathrm{~mm}$, para as folhas de aroeira.

Para avaliar a influência da temperatura no coeficiente de difusão efetivo, foi utilizada a equação de Arrhenius, descrita da seguinte forma:

$$
\mathrm{D}_{\mathrm{i}}=\mathrm{D}_{0} \exp \left(\frac{\mathrm{E}_{\mathrm{a}}}{\mathrm{RT}_{\mathrm{a}}}\right)
$$

em que $\mathrm{D}_{0}=$ fator pré-exponencial; $\mathrm{E}_{\mathrm{a}}=$ energia de ativação $\left(\mathrm{kJ} \mathrm{mol}^{-1}\right) ; \mathrm{R}=$ constante universal dos gases $\left(8,314 \mathrm{~kJ} \mathrm{kmol}^{-1} \mathrm{~K}^{-1}\right) ; \mathrm{T}_{\mathrm{a}}=$ temperatura absoluta $(\mathrm{K})$.

Os dados experimentais de cinética de secagem das folhas de aroeira foram submetidos à análise de regressão e seleção do modelo matemático adequado, para expressar a relação entre as variáveis estudadas. Para o ajuste dos modelos matemáticos aos dados experimentais, foi utilizado o programa computacional Statistica $7 \cdot 0^{\circledR}$.

Para análise do grau de ajuste de cada modelo, foram consideradas as magnitudes do coeficiente de determinação ajustado (variância explicada), do erro médio relativo e desvio padrão da estimativa, além da verificação do comportamento da distribuição dos resíduos.

Os valores de erro médio relativo $(\mathrm{P})$ e desvio padrão da estimativa (SE) foram calculados conforme descrito a seguir:

$$
\begin{aligned}
& P=\frac{100}{n} \sum_{i=1}^{n}\left(\frac{|Y-\hat{Y}|}{Y}\right) \\
& S E=\sqrt{\frac{\sum_{i=1}^{n}(Y-\hat{Y})^{2}}{G L R}}
\end{aligned}
$$

em que $\mathrm{n}=$ número de observações experimentais; $\mathrm{Y}=$ valor experimental; $\hat{\mathrm{Y}}=$ valor estimado pelo modelo; GLR = graus de liberdade do modelo.

\section{RESULTADOS E DISCUSSÃO}

Observou-se que, para todas as temperaturas utilizadas na secagem das folhas de aroeira, os modelos matemáticos ajustados aos dados experimentais apresentaram coeficientes de determinação $\left(\mathrm{R}^{2}\right)$ superiores a 97\% (Tabela 2), fato que, segundo Madamba et al. (1996) e Kashaninejad et al. (2007), indicam representação satisfatória destes modelos ao fenômeno de secagem estudado. Entretanto, a utilização do coeficiente de determinação como único critério de avaliação para a seleção dos modelos de secagem não constitui um bom parâmetro, sendo necessária a análise conjunta de outros parâmetros estatísticos (Mohapatra \& Rao 2005).

Constatou-se que boa parte dos modelos apresentou valores de erro médio relativo superiores a 10\% (Tabela 2), fato que, segundo Mohapatra \& Rao (2005), torna-os inadequados para a descrição de um determinado fenômeno, em especial o da secagem. Segundo Kashaninejad et al. (2007), os valores de erro médio relativo indicam desvio dos valores observados, em relação à curva estimada pelo modelo. Assim, conjuntamente a este parâmetro estatístico, optou-se por adotar, como critério adicional, o modelo ter bom ajuste para todas as temperaturas 
Tabela 2. Parâmetros estatísticos para os modelos de secagem das folhas de aroeira (Dourados, MS, 2013).

\begin{tabular}{|c|c|c|c|c|c|c|}
\hline \multirow{2}{*}{ Modelo } & \multicolumn{3}{|c|}{$40^{\circ} \mathrm{C}$} & \multicolumn{3}{|c|}{$50^{\circ} \mathrm{C}$} \\
\hline & SE (decimal) & $\mathrm{P}(\%)$ & $\mathrm{R}^{2}$ (decimal) & SE (decimal) & $\mathrm{P}(\%)$ & $\mathrm{R}^{2}$ (decimal) \\
\hline (2) & 0,0452 & 18,0100 & 0,9794 & 0,0446 & 39,0737 & 0,9784 \\
\hline (3) & 0,0310 & 10,3931 & 0,9909 & 0,0143 & 6,5099 & 0,9979 \\
\hline (4) & 0,0353 & 12,4517 & 0,9866 & 0,0328 & 26,6621 & 0,9876 \\
\hline (5) & 0,0094 & 3,3439 & 0,9993 & 0,0110 & 7,6447 & 0,9990 \\
\hline (6) & 0,0158 & 3,5700 & 0,9975 & 0,0138 & 7,0864 & 0,9979 \\
\hline (7) & 0,0135 & 4,8603 & 0,9983 & 0,0137 & 9,7979 & 0,9981 \\
\hline (8) & 0,0373 & 12,6233 & 0,9851 & 0,0340 & 25,6759 & 0,9866 \\
\hline (9) & 0,0438 & 18,0114 & 0,9794 & 0,0432 & 39,0760 & 0,9784 \\
\hline \multirow{2}{*}{ Modelo } & \multicolumn{3}{|c|}{$60^{\circ} \mathrm{C}$} & \multicolumn{3}{|c|}{$70^{\circ} \mathrm{C}$} \\
\hline & SE (decimal) & $\mathrm{P}(\%)$ & $\mathrm{R}^{2}$ (decimal) & SE (decimal) & $\mathrm{P}(\%)$ & $\mathrm{R}^{2}$ (decimal) \\
\hline (2) & 0,0582 & 55,6819 & 0,9758 & 0,0165 & 14,2744 & 0,9983 \\
\hline (3) & 0,0131 & 11,1969 & 0,9989 & 0,0172 & 13,3150 & 0,9985 \\
\hline (4) & 0,0247 & 26,6068 & 0,9951 & 0,0185 & 18,0396 & 0,9975 \\
\hline (5) & 0,0129 & 9,0764 & 0,9993 & 0,0078 & 3,9089 & 0,9998 \\
\hline (6) & 0,0139 & 12,7105 & 0,9986 & 0,0179 & 15,2170 & 0,9980 \\
\hline (7) & 0,0094 & 7,4390 & 0,9994 & 0,0115 & 6,4425 & 0,9993 \\
\hline (8) & 0,0207 & 19,3910 & 0,9965 & 0,0157 & 12,6484 & 0,9982 \\
\hline$(9)$ & 0,0548 & 55,6827 & 0,9758 & 0,0469 & 45,2582 & 0,9838 \\
\hline
\end{tabular}

estudadas, durante a secagem das folhas de aroeira. Desta forma, apenas os modelos de Henderson \& Pabis modificado e Midilli apresentaram magnitudes do erro médio relativo inferiores a $10 \%$, para todas as temperaturas do ar de secagem estudadas.

Além dos valores do coeficiente de determinação e do erro médio relativo, também foram calculados os valores do desvio padrão da estimativa (Tabela 2). Observou-se, em geral, que os modelos de Midilli e de Henderson \& Pabis modificado foram os que apresentaram as menores magnitudes de desvio padrão da estimativa, dentre todos os testados e para todas as temperaturas testadas (Tabela 2). De acordo com Draper \& Smith (1998), a capacidade de um modelo para descrever, com fidelidade, um determinado processo físico é inversamente proporcional ao valor do desvio padrão da estimativa. Desta forma, quanto menor for o valor desta variável, melhor será a qualidade de ajuste do modelo, em relação aos dados observados.

Assim, com base em todos os parâmetros estatísticos utilizados, recomendam-se os modelos de Hendesron \& Pabis modificado e de Midilli, para a representação do fenômeno de secagem das folhas de aroeira. Dentre eles, o de Midilli é, matematicamente, mais prático, apresentando menor número de parâmetros, tornando mais simples sua aplicação e uso, em simulações de secagem (Kashaninejad et al. 2007). Desta forma, nas condições em que este trabalho foi realizado, o modelo de Midilli foi selecionado para representar a secagem das folhas de aroeira.

Em trabalhos com outras espécies, como a carqueja (Radünz et al. 2011), folhas de fruta-de-lobo (Prates et al. 2012), Gundelia tourneforti L. (Evin 2012), folhas de capim-limão (Martinazzo et al. 2007) e folhas de manjericão (Reis et al. 2012), o modelo de Midilli também foi o que melhor se ajustou aos dados experimentais de secagem. O melhor ajuste do modelo de Midilli aos dados experimentais de secagem de plantas medicinais, provavelmente, está ligado à rápida perda de água, nos estádios iniciais do processo, neste tipo de produto, gerando uma curva de secagem mais acintosa e mais bem caracterizada, matematicamente, por este modelo.

Na Figura 1, são apresentadas, para as condições de secagem estudadas, comparações entre os valores observados e os estimados, pelo modelo de Midilli, da razão de umidade das folhas de aroeira, durante a secagem. Observou-se que, com o aumento da temperatura do ar de secagem, ocorreu redução no tempo necessário para a remoção de água do produto, fenômeno também observado por diversos pesquisadores, com outras espécies de plantas medicinais (Doymaz 2006, Martinazzo et al. 2007 e 2010, Premi et al. 2010, Radünz et al. 2011, Evin et al. 2012, Prates et al. 2012, Reis et al. 2012, Rocha et al. 2012).

Observou-se, ainda, que, para que as folhas de aroeira atingissem o teor de água de, aproximada- 


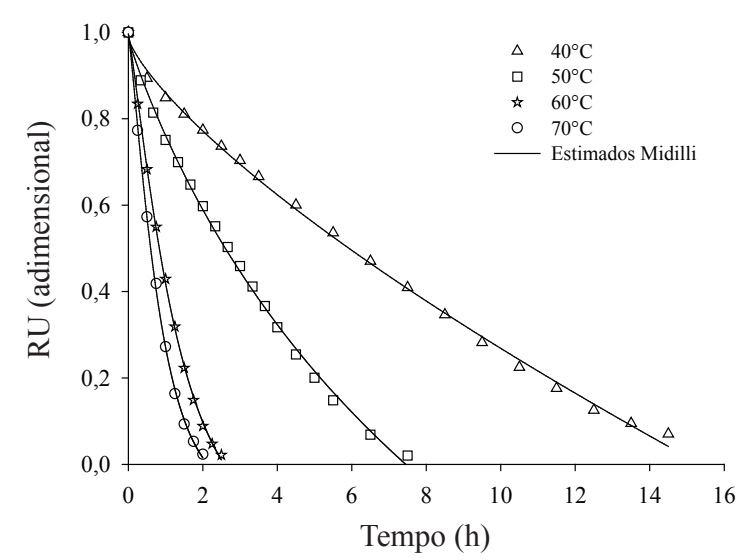

Figura 1. Valores experimentais e estimados, pelo modelo de Midilli, da razão de umidade de folhas de aroeira (Schinus terebinthifolius), durante a secagem (Dourados, MS, 2013).

mente, $10 \%$ (b.u.), foram necessárias 14,$7 ; 7,5 ; 2,5$; e 2,0 horas, respectivamente para as temperaturas de $40^{\circ} \mathrm{C}, 50^{\circ} \mathrm{C}, 60^{\circ} \mathrm{C}$ e $70^{\circ} \mathrm{C}$.

Evidenciou-se bom ajuste do modelo de Midilli aos dados observados (Figura 2), uma vez que estes ficaram próximos à linha de $45^{\circ}$, reforçando a aplicabilidade do modelo na predição dos dados de secagem das folhas de aroeira.

Com relação aos coeficientes do modelo de Midilli (Tabela 3), ajustados aos dados observados da cinética de secagem das folhas de aroeira, para as diferentes temperaturas do ar de secagem, observou-se que somente os parâmetros " $k$ " e " $n$ " do modelo de Midilli apresentaram tendência definida na variação da magnitude de seus valores com a temperatura do ar de secagem. Com a elevação da temperatura do ar de secagem, houve aumento nos valores encontrados para os parâmetros " $k$ " e " $n$ ", sendo que, para os parâmetros "a" e "b", não houve tendência observada. As variações dos parâmetros "a" e "b" se devem mais a ajustes matemáticos do que relacionados a algum fenômeno de secagem,

Tabela 3. Parâmetros do modelo de Midilli, para diferentes temperaturas de secagem de folhas de aroeira (Dourados, MS, 2013).

\begin{tabular}{ccccc}
\hline Temperatura $\left({ }^{\circ} \mathrm{C}\right)$ & $\mathrm{a}$ & $\mathrm{k}$ & $\mathrm{n}$ & $\mathrm{b}$ \\
\hline 40 & 0,9893 & 0,1043 & 0,7038 & $-0,0315$ \\
50 & 0,9876 & 0,2151 & 0,9062 & $-0,0351$ \\
60 & 0,9948 & 0,7836 & 1,1804 & $-0,0352$ \\
70 & 0,9964 & 1,2208 & 1,1925 & $-0,0228$ \\
\hline
\end{tabular}

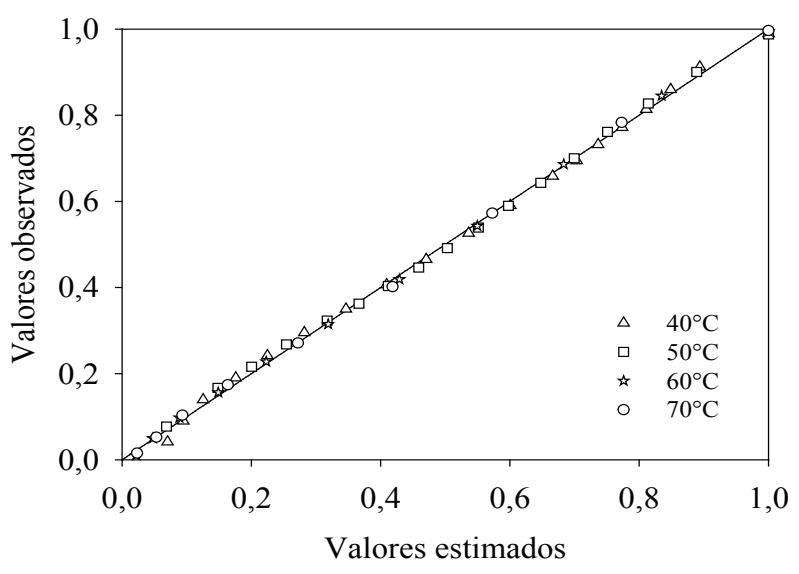

Figura 2. Valores de razão de umidade observados e estimados pelo modelo de Midilli, para a secagem de folhas de aroeira (Schinus terebinthifolius) (Dourados, MS, 2013).

uma vez que o modelo de Midilli é semiempírico (Midilli et al. 2002).

A constante de secagem $(\mathrm{k})$, que representa as condições externas de secagem, pode ser utilizada como uma aproximação para caracterizar o efeito da temperatura, e está relacionada com a difusividade efetiva no processo de secagem, no período decrescente, e à difusão líquida que controla o processo (Babalis \& Belessiotis 2004).

A relação dos parâmetros " $k$ " e " $n$ " do modelo de Midilli, em função da temperatura do ar de secagem das folhas de aroeira, pode ser descrita, respectivamente, pelas seguintes equações lineares:

$$
\begin{aligned}
& \mathrm{K}=0,0392 \mathrm{~T}-1,5740\left(\mathrm{R}^{2}=0,9458\right) \\
& \mathrm{n}=0,0174 \mathrm{~T}+0,0386\left(\mathrm{R}^{2}=0,9119\right)
\end{aligned}
$$

Observa-se, nas Equações 14 e 15, elevadas magnitudes do coeficiente de determinação $\left(\mathrm{R}^{2}\right)$, referentes às equações utilizadas para descrever os valores dos parâmetros "k" e " $n$ " do modelo de Midilli, em função da temperatura do ar. Utilizando-se estas equações, juntamente com a média dos valores dos parâmetros "a" e "b", para todas as condições do ar de secagem, tem-se a aplicabilidade de se poder estimar o teor de água das folhas de aroeira em função do tempo e da temperatura do ar de secagem, por meio da seguinte expressão:

$$
\begin{aligned}
& \mathrm{M}=\mathrm{M}_{\mathrm{e}}+\left(\mathrm{M}_{\mathrm{i}}-\mathrm{M}_{\mathrm{e}}\right)\{0,9920 \exp [-(0,0392 \mathrm{~T}- \\
& \left.\left.1,5740) \theta^{(-0,0174 \mathrm{~T}+0,0386)}\right]-0,0312 \theta\right\} \\
& \text { em que } \mathrm{T}=\text { temperatura }\left({ }^{\circ} \mathrm{C}\right) ; \theta=\text { tempo }(\mathrm{h}) .
\end{aligned}
$$


$\mathrm{Na}$ Tabela 4, são apresentados os valores médios do coeficiente de difusão efetivo obtidos durante a secagem das folhas de aroeira, para diferentes condições de temperatura do ar, considerando-se a espessura de 0,3631 mm. Notou-se que os valores do coeficiente de difusão efetivo aumentaram com o incremento da temperatura do ar de secagem. Quando há aumento na temperatura, a viscosidade da água diminui e, sendo a viscosidade uma medida da resistência do fluído ao escoamento, variações desta propriedade implicam em alterações na difusão da água nos capilares das folhas de aroeira, de modo a favorecer a movimentação deste fluído no produto. Outro fator que pode ser acrescentado a esta variação do coeficiente de difusão efetivo é que, com a elevação da temperatura, aumenta-se o nível de vibração das moléculas de água, o que, também, contribui para a celeridade da difusão (Goneli et al. 2007).

De acordo com Rizvi (1995), o coeficiente de difusão efetivo é dependente da temperatura do ar de secagem, além da variedade e composição dos materiais, dentre outros, o que justifica o seu aumento, com incrementos da temperatura do ar de secagem.

A variação entre os valores do coeficiente de difusão ficaram entre $0,1476 \times 10^{-11} \mathrm{~m}^{2} \mathrm{~s}^{-1} \mathrm{e}$ $1,5811 \times 10^{-11} \mathrm{~m}^{2} \mathrm{~s}^{-1}$, para a aroeira, na faixa de temperatura de $40-70^{\circ} \mathrm{C}$ (Tabela 4). Os valores do coeficiente de difusão efetivo calculados para folhas de aroeira estão coerentes com os relatados na literatura, para secagem de produtos agrícolas, que, de acordo com Madamba et al. (1996), apresentam-se na ordem de $10^{-9} \mathrm{~m}^{2} \mathrm{~s}^{-1}$ a $10^{-11} \mathrm{~m}^{2} \mathrm{~s}^{-1}$.

A dependência do coeficiente de difusão efetivo, com relação à temperatura do ar de secagem, tem sido, satisfatoriamente, descrita pela equação de Arrhenius (Corrêa et al. 2007, Goneli et al. 2007, Martinazzo et al. 2007, Resende et al. 2007). Têm-se, na Figura 3, os valores de Ln (D), em função do

Tabela 4. Coeficiente de difusão efetivo, durante a secagem de folhas de aroeira, em diferentes temperaturas (Dourados, MS, 2013).

\begin{tabular}{cc}
\hline Temperatura $\left({ }^{\circ} \mathrm{C}\right)$ & $\mathrm{D} \times 10^{-11}\left(\mathrm{~m}^{2} \mathrm{~s}^{-1}\right)$ \\
\hline 40 & 0,1476 \\
50 & 0,3265 \\
60 & 1,1429 \\
70 & 1,5811 \\
\hline
\end{tabular}

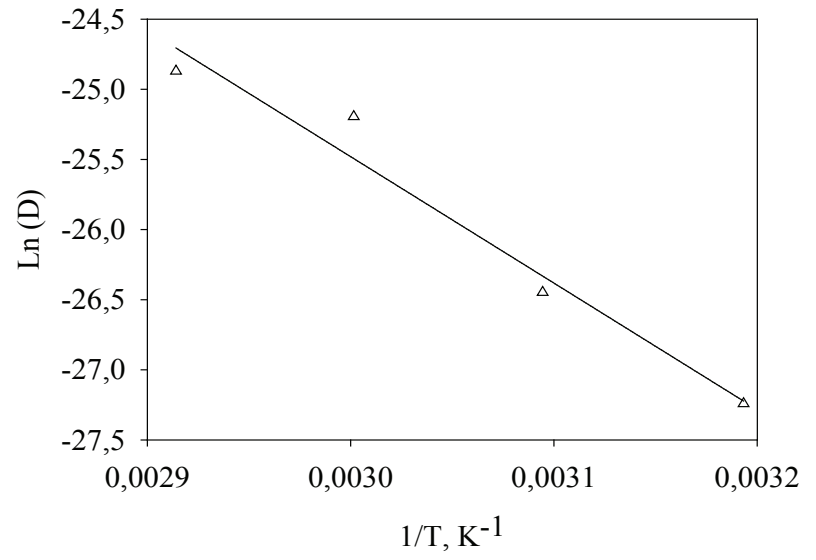

Figura 3. Representação de Arrhenius para o coeficiente de difusão efetivo, em função da temperatura do ar, durante a secagem de folhas de aroeira (Dourados, MS, 2013).

inverso da temperatura absoluta $\left(\mathrm{K}^{-1}\right)$, obtidos para as folhas de aroeira.

A inclinação da curva da representação de Arrhenius fornece a relação $\mathrm{Ea} / \mathrm{R}$, enquanto a sua interseção com o eixo das ordenadas indica o valor de $\mathrm{D}_{\text {. }}$ A Equação 17 apresenta o coeficiente da equação de Arrhenius, ajustada para os coeficientes de difusão efetivos das folhas de aroeira, calculado de acordo com a Equação 11:

$$
\mathrm{k}=4,8040 \exp \left(-\frac{74.963,7130}{\mathrm{R} \mathrm{T}_{\mathrm{a}}}\right)
$$

Como pode ser observado na Equação 17, a energia de ativação para a difusão da água, durante a secagem das folhas de aroeira, foi de $74,96 \mathrm{~kJ} \mathrm{~mol}^{-1}$. Este valor difere dos encontrados por Martinazzo et al. (2007), para folhas de capim-limão $\left(63,47 \mathrm{~kJ} \mathrm{~mol}^{-1}\right)$, e Doymaz (2006), para folhas de Mentha spicata L. $\left(62,96 \mathrm{~kJ} \mathrm{~mol}^{-1}\right)$, enquanto se aproxima dos observados por Rocha et al. (2012), durante a secagem de tomillo $\left(77,16 \mathrm{~kJ} \mathrm{~mol}^{-1}\right)$.

Nos processos de secagem, quanto menor a energia de ativação, maior será a difusividade de água no produto. A energia de ativação é uma barreira que deve ser ultrapassada, para que o processo de difusão possa ser desencadeado no produto (Kashaninejad et al. 2007). Segundo Zogzas et al. (1996), a energia de ativação, para produtos agrícolas, varia entre $12,7 \mathrm{~kJ} \mathrm{~mol}^{-1}$ e $110 \mathrm{~kJ} \mathrm{~mol}^{-1}$, estando a energia de ativação encontrada no presente trabalho dentro da faixa proposta por estes autores. 


\section{CONCLUSÕES}

1. Os modelos matemáticos de Henderson \& Pabis modificado e Midilli foram os que apresentaram melhores ajustes estatísticos aos dados experimentais da cinética de secagem, sendo o modelo de Midilli selecionado para a representação da secagem das folhas de aroeira devido à sua maior simplicidade.

2. O aumento da temperatura do ar de secagem promoveu redução no tempo necessário para a remoção de água do produto, durante a secagem.

3. O coeficiente de difusão efetivo aumentou com a elevação da temperatura do ar de secagem, e esta relação pode ser descrita pela equação de Arrhenius, que apresentou energia de ativação para a difusão líquida, durante a secagem, de $74,96 \mathrm{~kJ} \mathrm{~mol}^{-1}$, para as folhas de aroeira.

\section{REFERÊNCIAS}

AMERICAN SOCIETY OF AGRICULTURAL AND BIOLOGICAL ENGINEERS (ASABE). Moisture measurement: forages: standard S358.2 DEC1988, R2008. In: ASABE. Standards, engineering practices, and data. St. Joseph: ASABE, 2010. p. 684-685.

BABALIS, S. J.; BELESSIOTIS, V. G. Influence of the drying conditions on the drying constants and moisture diffusivity during the thin-layer drying of figs. Journal of Food Engineering, Londres, v. 65, n. 3, p. 449-458, 2004.

BERBERT, P. A. et al. Simulation of coffee drying in a fixed bed with periodic airflow reversal. Journal of Agricultural Engineering Research, Londres, v. 60, n. 3, p. 167-173, 1995.

CORRÊA, P. C. et al. Modelagem matemática para a descrição do processo de secagem do feijão (Phaseolus vulgaris L.) em camadas delgadas. Engenharia Agrícola, Jaboticabal, v. 27, n. 2, p. 501-507, 2007.

DOYMAZ, I. Thin-layer drying behaviour of mint leaves. Journal of Food Engineering, Londres, v. 74, n. 3 , p. 370-375, 2006.

DRAPER, N. R.; SMITH, H. Applied regression analysis. 3. ed. New York: John Wiley \& Sons, 1998.

EVIN, D. Thin layer drying of Gundelia tournefortii L. Food and Bioproducts Processing, Melbourne, v. 90, n. 2, p. 323-332, 2012.

GONELI, A. L. D. et al. Estudo da difusão de umidade em grãos de trigo durante a secagem. Ciência e Tecnologia de Alimentos, Campinas, v. 27, n. 1, p. 135-140, 2007.
KASHANINEJAD, M. et al. Thin-layer drying characteristics and modeling of pistachio nuts. Journal of Food Engineering, Londres, v. 78, n. 1, p. 98-108, 2007.

KEEY, R. B. Drying: principles and practice. New York: Pergamon Press, 1972.

LORENZI, H.; MATOS, F. J. A. Plantas medicinais no Brasil: nativas e exóticas. Nova Odessa: Plantarum, 2002.

MADAMBA, P. S.; DRISCOLL, R. H.; BUCKLE, K. A. Enthalpy-entropy compensation models for sorption and browning of garlic. Journal of Food Engineering, Londres, v. 28, n. 1, p. 109-119, 1996.

MARTINAZZO, A. P. et al. Análise e descrição matemática da cinética de secagem de folhas de capim-limão. Revista Brasileira de Engenharia Agrícola e Ambiental, Campina Grande, v. 11, n. 3, p. 301-306, 2007.

MARTINAZZO, A. P. et al. Modelagem matemática e parâmetros qualitativos da secagem de folhas de capimlimão [Cymbopogon citratus (DC.) Stapf]. Revista Brasileira de Plantas Medicinais, Botucatu, v. 12, n. 4, p. 488-498, 2010.

MIDILLI, A.; KUCUK, H.; YAPAR, Z. A. New model for single-layer drying. Drying Technology, New York, v. 20, n. 7, p. 1503-1513, 2002.

MIGUEL, M. D.; MIGUEL, O. G. Desenvolvimento de fitoterápicos. 2. ed. Ribeirão Preto: Tecmed, 2004.

MOHAPATRA, D.; RAO, P. S. A thin layer drying model of parboiled wheat. Journal of Food Engineering, Londres, v. 66, n. 4, p. 513-518, 2005.

OLIVEIRA, M. T. R. et al. Avaliação de modelos matemáticos na descrição das curvas de secagem por convecção de Pectis brevipedunculata (Gardner) Sch. Bip. Revista Brasileira de Plantas Medicinais, Botucatu, v. 15, n. 1, p. 1-12, 2013.

OZDEMIR, M.; DEVRES, Y. O. The thin layer drying characteristics of hazelnuts during roasting. Journal of Food Engineering, Londres, v. 42, n. 4, p. 225-233, 1999.

PANCHARIYA, P. C.; POPOVIC, D.; SHARMA, A. L. Thin-layer modeling of black tea drying process. Journal of Food Engineering, Londres, v. 52, n. 4, p. 349-357, 2002.

PARK, K. J.; VOHNIKOVA, Z.; BROD, F. P. R. Evaluation of drying parameters and desorption isotherms of garden mint leaves (Mentha crispa L.). Journal of Food Engineering, Londres, v. 51, n. 3, p. 193-199, 2002.

PRATES, M. F. O. et al. Cinética de secagem de folhas de Solanum lycocarpum A. St.-Hil. (fruta-de-lobo). Revista Brasileira de Plantas Medicinais, Botucatu, v. 14, n. 3, p. 514-521, 2012. 
PREMI, M. et al. Kinetics of drumstick leaves (Moringa oleifera) during convective drying. African Journal of Plant Science, Pretoria, v. 4, n. 10, p. 391-400, 2010.

RADÜNZ, L. L. et al. Avaliação da cinética de secagem de carqueja. Engenharia na Agricultura, Viçosa, v. 19, n. 1, p. 19-27, 2011.

REIS, R. C. et al. Cinética de secagem de folhas de manjericão (Ocimus basilicum L.) via infravermelho. Revista Brasileira de Engenharia Agrícola e Ambiental, Campina Grande, v. 16, n. 12, p. 1346-1352, 2012.

RESENDE, O. et al. Bean moisture diffusivity and drying kinetics: a comparison of the liquid diffusion model when taking into account and neglecting grain shrinkage. Spanish Journal of Agricultural Research, Madri, v. 5, n. 1, p. 51-58, 2007.

RIZVI, S. S. H. Thermodynamic properties of foods in dehydration. In: RAO, M. A.; RIZVI, S. S. H. Engineering properties of foods. New York: Academic Press, 1995. p. 223-309.

ROCA, E. et al. Effective moisture diffusivity modeling food structure and hygroscopicity. Food Chemistry, Reading, v. 106, n. 4, p. 1428-1437, 2008.

ROCHA, R. P. et al. Cinética del secado de tomillo. Revista Brasileira de Engenharia Agrícola e Ambiental, Campina Grande, v. 16, n. 6, p. 675-683, 2012.

SOYSAL, Y.; ÖZTEKIN, S. Equilibrium moisture content equations for some medicinal and aromatic plants. Journal of Agricultural Engineering Research, Londres, v. 74, n. 3, p. 317-324, 1999.

ZOGZAS, N. P.; MAROULIS, Z. B.; MARINOSKOURIS, D. Moisture diffusivity data compilation in foodstuffs. Drying Technology, New York, v. 14, n. 10, p. 2225-2253, 1996. 